\title{
Transaction Based Business Process Modeling
}

\author{
Frantisek Hunka \\ University of Ostrava, Faculty of Science, \\ Dvorakova 7, 70103 Ostrava, \\ Czech Republic \\ Email: frantisek.hunka@osu.cz
}

\begin{abstract}
A term of transaction which has its origin in database processing, representing a set of operations that must be performed all or none of them. The notion of transaction is also used in some business process modeling approaches such as the DEMO (Design \& Engineering Methodology for Organizations) and the REA (resource-event-agent) value modeling approach. The DEMO's transaction forms a basic building block from which a business process is composed. The REA value modeling approach utilizes transactions in an REA model representing a business process. In general, both methodologies utilize the notion of transaction, which has, however, a different meaning in these approaches. The aim of the paper is to describe the basic models of both approaches and to show, with the 'rent-a-car' example, the principal differences between the notions of transaction between them. The paper also reflects on possible mutual collaboration between both approaches.
\end{abstract}

\section{INTRODUCTION}

$\mathrm{B}$ USINESS process modeling unquestionably belongs to software development process. It usually directly influences the database solution of the problem domain. Most of the business process modeling methodologies simply originated form 'best practice' without a vigorous theory from which the methodology is derived, see [9]. They mostly focus on production actions, which are usually described as an event that happens instantaneously or over a period of time. Generally, the most recommended notation of business processes is an UML activity diagram with swim lines, see [8]. Each swim line represents a human being, more precisely an actor role. Human beings are an inseparable part of business process modeling. However, the absence of a vigorous theory means that business process modeling approaches suffer from various incompleteness.

DEMO methodology stems from Enterprise Ontology [3] which represents a generic approach to business process modeling. The benefit of this methodology is that it perfectly identifies principal transactions that create the business process including human beings. It also provides necessary abstractions that enable us to obtain the essence of the modeling reality. On the other hand, this methodology is designed to be generic, which means that it registers production activities and is aware of them but without affecting them.

The other ontology which is at the core of our interest is REA ontology, see [1]. Its name is derived from three fundamental concepts, namely: Resources, Events and Agents. This modeling approach originated from accountancy systems but was developed

\author{
Roman Belunek \\ University of Ostrava, Faculty of Science, \\ Dvorakova 7, 70103 Ostrava, \\ Czech Republic \\ Email: Roman.Belunek@osu.cz
}

into a fully-fledged tool for business process modeling. Economic resources are things of economic value that have utility for economic agents and for that reason they are planned, monitored, and controlled. Examples of economic resources are money, raw materials, labor, tools, products, and services. Economic events are activities within an enterprise that represent either an increment or a decrement in the value of economic resources. Some economic events occur instantaneously, some occur over time. Examples of economic events are sales of goods, rentals, and provision and use of services. Economic agents are individuals or organizations that participate in the control and execution of economic events. Examples of economic agents are customers, vendors, employees and enterprises. The structure of the paper is as follows: Section Two describes the main features of the DEMO methodology that are further utilized. The REA value modeling approach is clarified in Section Three. After a narrative description of the example, Section Four states the DEMO and REA solution to the example. Section Five discusses both approaches and Section Six summarizes the results achieved.

\section{DEMO Methodology}

According to DEMO methodology [3], [4] an organization is composed of people (social individuals) that perform two kinds of acts, production acts and coordination acts. By performing production acts, people fulfill the aims of the organization. A production act can be either material or immaterial. By a material production act we mean a tangible act such as a manufacturing or transportation act. By an immaterial act we mean an intangible act such as the approval of an insurance claim or delivery of a judgment. By performing coordination acts human beings enter into and comply with commitments. They initiate and coordinate production acts. Abstracting from the particular subject that performs the action, the notion of the actor role is introduced. A subject in his/her fulfillment of an actor role is called an actor.

The result of successfully performing a production act is a production fact. An example of a production fact may be that the payment has been paid or an offered service was accepted. All realization issues are fully abstracted. Only the facts as such are relevant, not how they are achieved. The result of successfully performing a coordination act is a coordination fact. Examples of coordination acts are requesting and promising a production fact.

The diagram in Fig. 1 shows the standard transaction pattern (transaction). It contains two actor roles, the initiator and the executor and coordination and production acts between them. Each transaction starts with a request coordination act made by the initiator. In response to the request, the executor performs either a promise or decline coordination act. In short, a decline means the 
end of a transaction. The promise goes on in a production act which results in a production fact. The production fact is stated to the initiator who can either accept it or reject it. The standard transaction pattern can be extended to the complete transaction pattern. In this case, the transaction pattern also contains four cancellation patterns that enable revoking of an act and completely model real conditions. For the purpose of the paper, only the transaction pattern will be used. The transaction itself can be expressed in a more condensed way, see Fig. 2.

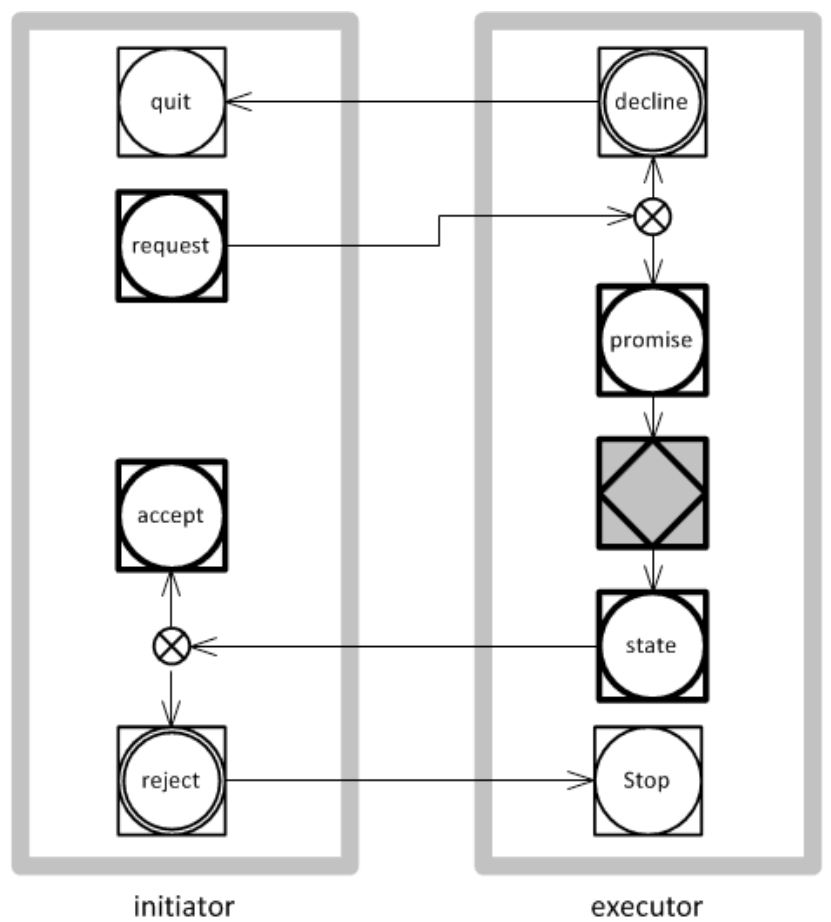

Fig. 1 DEMO transaction - standard pattern Source: [3]

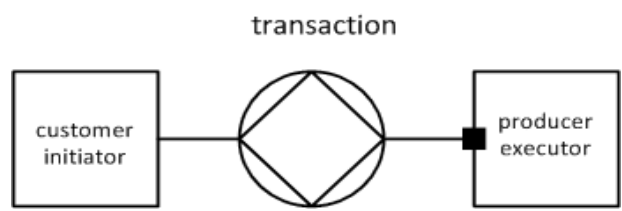

Fig. 2 Transaction in the Construction Model Source: [3]

The diagram in Fig. 2 shows the relation between the initiator (the relation is indicated by the plain line) and the executor (the relation is indicated by the dot at the executor). The DEMO methodology provides four mutually integrated aspect models. In this paper, only the Construction Model is used. The commission of the Construction Model is to identify actor roles and transactions. The task of the Process Model is to show how transactions are causally and conditionally related. The Construction Model has a crucial position in comparison with the REA model. The basic unit for declaring business processes is the notion of transaction. Transaction represents the basic building block between two social subjects. Apart from the basic states of request, promise, production, state and accept it also contains the other states that address erroneous states such as decline and refuse and the states which come from cancellation. Business process in DEMO methodology is defined as a set of enclosing transactions with a definite result (fact).

\section{REA Value Modeling Approach}

The main benefits of the REA approach are being able to keep track of primary and raw data about economic resources. This explains why the REA approach offers a wider, more precise, and more up-to-date range of reports. All accounting artifacts such as debit, credit, journals, ledgers, receivables, and account balances are derived from data describing exchange and conversion REA processes. All reports based on accounting artifacts are always consistent, because they are derived from the same data by [6]. For example, data describing a sale event is used in warehouse management, payroll, distribution, finance and other application areas, without transformations or adjustments. REA ontology also benefits from the presence of a semantic and application independent data model, an object oriented perspective, and abstraction from technical and implementation details. These features enable the possibility of calculating the value of the enterprise's resources on demand, as opposed to calculation at predetermined intervals.

Apart from keeping track of the past and current economic events, an REA model has the capability of modeling economic events that will occur in the future. However, the principal feature of REA ontology that originates from accountancy systems is that it explicitly distinguishes between past and current events and events performed in the future. And thus for events that occur in the future, REA introduces a new entity called a commitment entity. For this reason, the utilization of a commitment entity is not obligatory but depends only on the specific modeling context. That is, if the model does not address 'future events' there is no need for modeling a commitment entity. In addition, in many cases, only the data concerning real production, which means data about economic events and directly related entities (economic resources, economic agents), are utilized for further processing. The operational level is the part of the REA model which deals with the past and current events. The policy level is the upper part of the REA model which addresses the future events for which the commitment entity and contract entity are necessary. Although the operational level of the REA model can exist independently, the policy level of the REA model can exist only when mutually bound with the operational level. An REA model is illustrated in Fig. 3.

The commitment entity addresses the issue of modeling promises of the future economic events and the issue of the resources reservation, see [5]. Commitment entities and their relationships with other entities are shown in Fig. 3. This figure shows that the commitment entity copies the structure of the event entity to a considerable extent, by which we mean the existence of an increment and decrement commitment and exchange reciprocity relationship.The relationship of committed provide and committed receive means that some level of agreement about the future exchange must be achieved between economic agents. The exchange reciprocity relationship between the increment and decrement commitments identifies which resources are promised to be exchanged for which others. The reciprocity relationship is a relation of many-to-many $\left(1 . .^{*}, 1 .{ }^{*}\right)$, see [6]. 


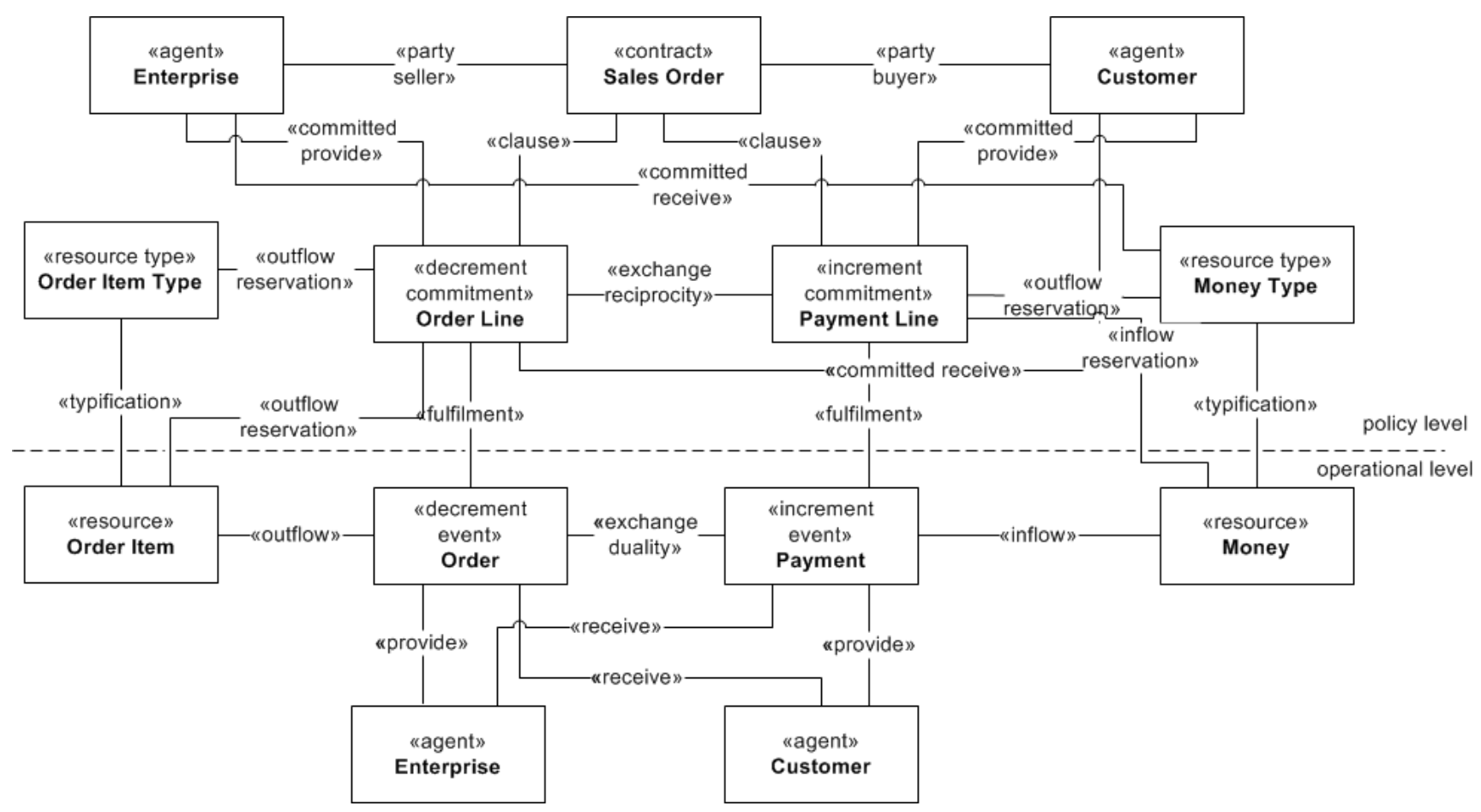

Fig. 3 REA model of an exchange process Source: adapted from [6]

Each commitment is related to an economic resource by a reservation relationship, which specifies which resources will be needed or expected by future economic events. The reservation relationship between the resource and commitment entities represents the obligation of economic agents to provide or receive rights to economic resources in exchange processes and represents scheduled usage, consumption or production of economic resources in conversion processes.

In its basic form, an REA transaction is represented by an economic event, an economic resource and a pair of economic agents. The economic event represents an event that happens or has happened in the past. REA transactions are related to each other by a duality relationship which is located at the operational level. In order to model future economic events, an REA model has to be extended with the policy level. This level contains commitment entities, contract entities and resource type entities. In short, a resource type entity represents a category item whereas a resource entity represents a physical item.

\section{RENT-A-CAR EXAMPLE}

This practical and probably familiar example can elucidate the differences and common issues of both methodologies. The Rent-acar example covers both current and future events and it is not too complex to comprehend. To introduce the problem a short narrative description follows. Rent-a-car is a service which is provided either to walk-in customers or customers who make a rental reservation by telephone, fax or email. A car may be rented on the same day or may be reserved for a specific term in the future after a contract between an employee of the rental company and a customer has been signed. The company which rents out cars has many branches around the country. So the rented car may be picked up and dropped of at different branches. The rental payment depends directly on the number of days of rental and kind of car rented. The signed contrast states, among other things, the period of the rental and the name of the branch where the car will be dropped off. If the period of rental or/and the drop off branch do not coincide with the conditions in the signed contract, the customer is liable for a penalty payment. The contracted payment must be made by the starting day of the rental at the latest. Additional penalty payments must be made at the drop off point.

\section{DEMO Solution}

This solution comprises the Construction model as it is fully in compliance with the necessity of both approaches comparison. The Construction model requires identification of the actor roles, transaction kinds and product kinds. In terms of actor roles there is an actor role CA0 who represents an employee of the rental company. The Construction model is illustrated in Fig. 4.

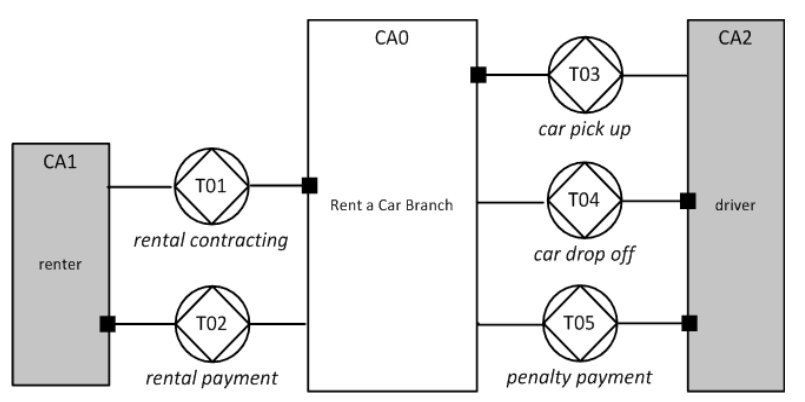

Fig. 4 Demo Construction model Source: [4] 
The other actor roles represent a renter and a driver respectively. Utilizing the actor role principle provides a more accurate modeling perspective because a human being can have more than one actor role and one actor role can be represented by different human beings.As it is not a complex example, it is not difficult to identify essential transactions. The first transaction T01 - rental contracting covers signing a contract to rent a car. The next transaction T02 - rental payment must be promised before the contract is signed and this must be done by the first day of the rental at the latest. The car pick-up transaction (T03) includes the promise of the rental company employee regarding the starting conditions of the rental. The other part of this transaction covers the pick up of the car. The car drop-off transaction (T04) is composed of two parts. In the first part the customer promises to observe the conditions specified in the contract. The second part of the transaction represents the actual drop-off the car at a branch of the rental company. The penalty payment transaction (T05) is an optional transaction which is executed if the driver exceeds conditions agreed in the contract.

Going more carefully through the transactions it can observe that coordination steps (act \& fact) enable mutual interconnecting (enclosing) of the transactions. The rental contracting transaction (T01) includes entering into and complying with a mutual commitment regarding the transaction T03, the car pick-up and transaction T04, the car drop-off. Both transactions involve detailed specifications about the beginning and end of the rental in the form of commitments. From the narrative description it is clear that the T02 rental payment transaction must be committed before the signing of the rental contract. This is due to the fact that in the text description the renter has to complete the payment by the first day of the committed car rental at the latest. The renter and the employee of the branch must also enter into and comply with any penalty payment to be paid if the period of rental is exceeded or the car is not returned to the branch where it should be dropped off. From this analysis it follows that at the rental contracting stage only the T01, rental contracting transaction, is accepted. The rental payment transaction (T02) must be paid by the starting day of the rental at the latest. The other transactions are usually promised but not yet accepted. The T03 car pick-up transaction is accepted only on the day that the rental starts.

As can be seen from the example, DEMO strictly follows actual conditions and provides great flexibility for a true description of a modelled reality. For example, in some cases the rental payment can be paid at the end of the rental or an advance payment must be made before the rental. This ability of DEMO is enabled by the distinction between the coordination and production acts and fact in a transaction. DEMO methodology is also beneficial when an immaterial product such as a judgement or a schedule is created within the transaction.

\section{REA Solution}

An REA solution can be modelled in two variations. The first variation represents the operational level of the REA model, whereas the second variation stands for both the operational and policy levels. The first variation enables the modeling of current and past economic events, whereas the second variation also enables the modeling of past, current and future events, described in [2]. Economic events, as a basic part of the REA transactions, must be identified first. Next, the corresponding economic resource and economic agents must be found. Once this has been done, a duality relationship between economic events must be established. The REA value modeling approach usually works with at least one pair of transactions which must be complementary to each other (give and take).

The first REA transaction is created by the rent-a-car economic event, the rent-a-car service economic resource and the renter and rental car company economic agents. The renter agent receives the rental service for a given period of time and the rental car company provides the car. The second transaction is formed by the rental payment economic event, the money economic resource and the pair of economic agents, the same as in the first transaction. What differs is the relationships between the agents and the events. In this case, the rental agent provides the money resource and the rental car company receives the money resource. The third transaction is similar to the second transaction apart from an additional event, which is the penalty payment economic event. These three transactions are related to each other by a duality relationship. This relationship allows us to keep track of which resources have been exchanged for which others.

The REA model illustrated in Fig. 6 represents the second variation of an REA model which includes future events. These events are in the REA approach represented by the commitment entities. The contract entity is composed of the commitment entities and terms. Under the conditions specified by the terms, a contract can create additional commitments. Thus, the contract can specify what should happen if the commitments are not fulfilled. Economic agents are related to the contract entity by the party relationship.

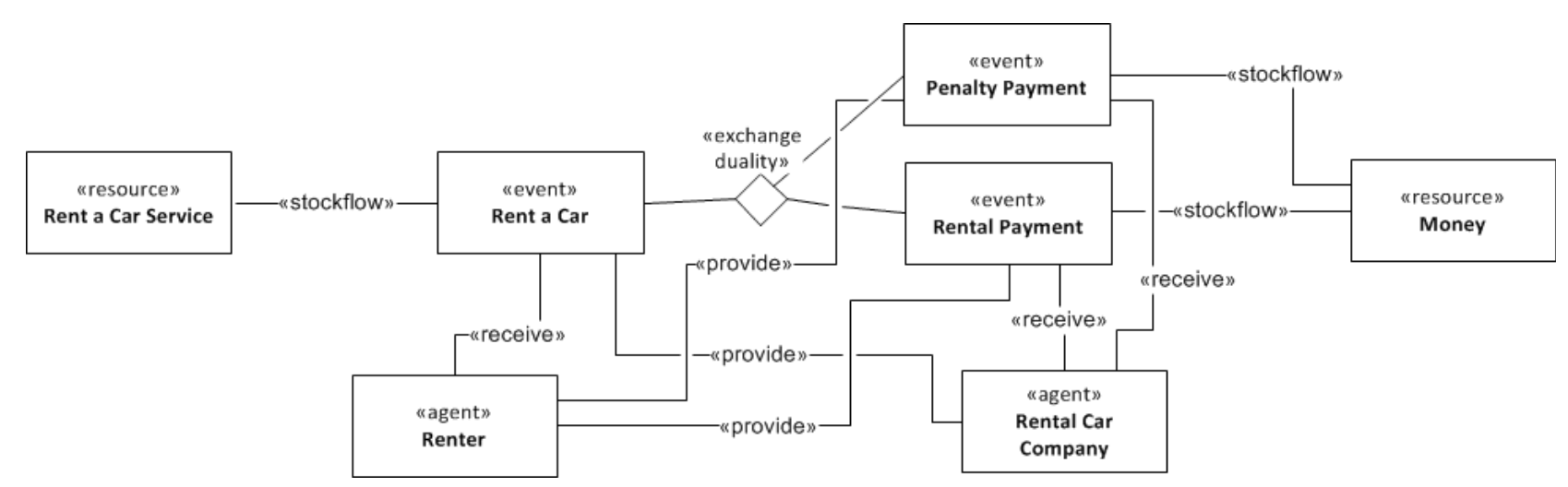

Fig. 5 REA model - operational level (first variation) Source: authors 


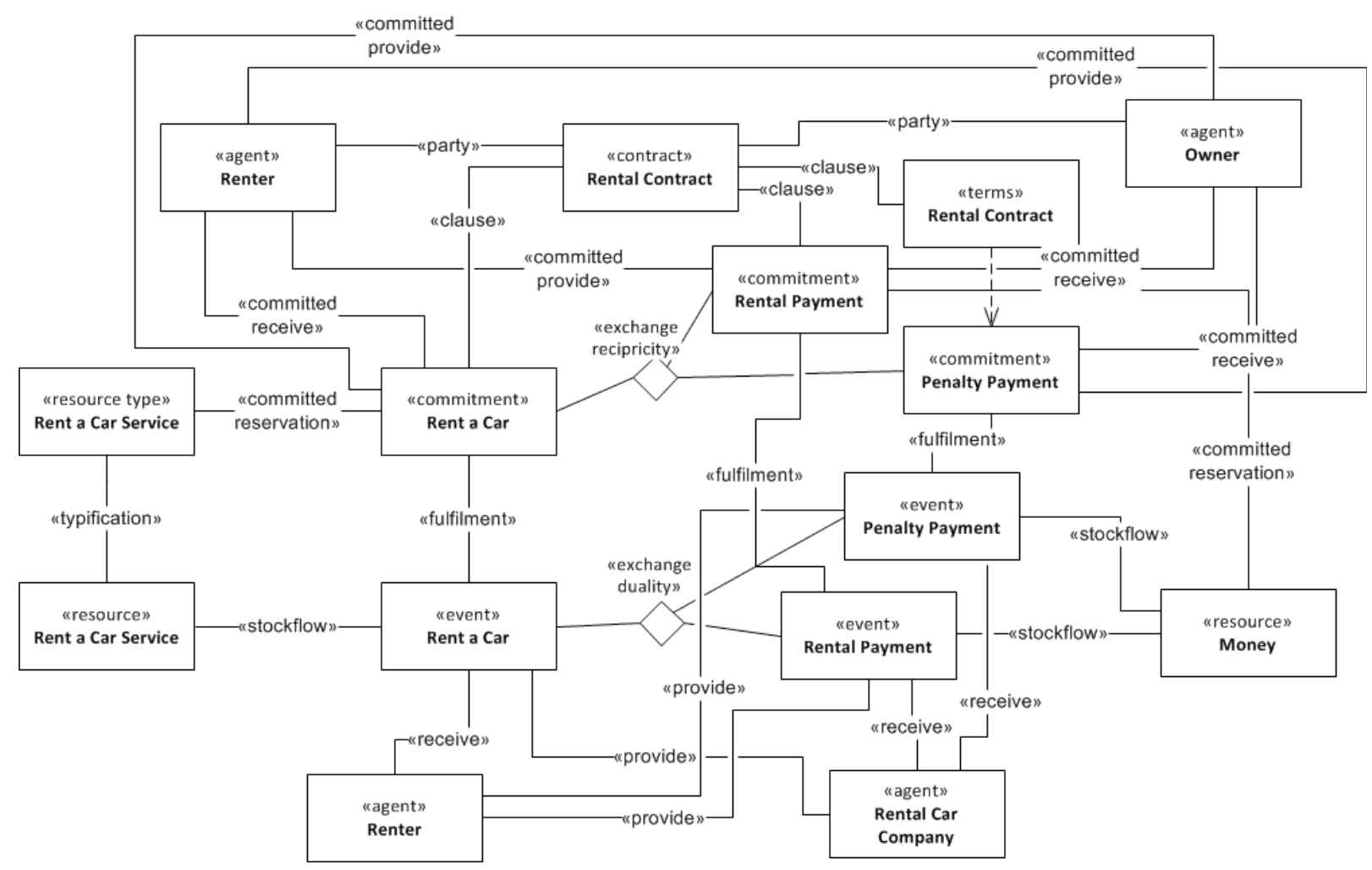

Fig. 6 REA model (second variation) Source: authors

The structure of the commitment entities corresponds with the structure of the event entities. The commitment entities are related to each other by a reciprocal relationship. The practical meaning of the reciprocal relationship is that the future events have been promised and that the resources have been reserved for the future events. The REA solution copies the domain rules.

\section{Discussion}

Although, the DEMO transaction and the REA transaction have similar formal meaning their semantic representations are significantly different. This is caused by the fact that the DEMO methodology represents a generic ontology, whereas the REA modeling approach stands for a domain-specific ontology. DEMO methodology utilizes a 'single' transaction as a basic building block from which a business process is composed. The operation axiom divides actions into coordination and production actions and each action is concluded by the result (fact). The DEMO transaction model contains cancellation and revoking operations and is far the most suitable for modeling real world. The DEMO transaction integrates past, current, and future events into one consistent unit. Transactions in a business process are organized in a tree structure, which is far closer to a domain model than a flat sequential structure used by other business process methodologies, including the REA modeling approach.

Among others, it is the operation axiom that has brought DEMO far closer to the world of human beings. Coordination actions enable human beings to enter into and comply with commitments. A production fact comes into existence after it is accepted by the corresponding coordination fact. Being a generic ontology, DEMO knows about real world events with good empirical evidence but it has limited means to deal with production acts and facts.

Contrary to DEMO methodology, the REA value modeling approach does not distinguish between coordination and production activities. Above all, this modeling approach keeps track of the value of economic resources. These resources can either be exchanged for other economic resources or be converted to different economic resources. The REA model, which represents a business process, is composed of at least two mutually binding transactions representing 'give' and 'take' operations in an exchange process, or 'use', 'consume' or 'produce' operations in a conversion process. In an analogy with the DEMO building block, the REA building block is a structure that connects at least two semantically bound transactions. By semantically it is meant that the binding transactions are in compliance with economic laws for the exchange or conversion of economic resources. Despite the benefits of the REA approach which were summarized in the introductory section, there are several drawbacks which prevent this ontology from becoming more widespread. Among REA drawbacks is the explicit separation of past and current events and future events [7]. In the REA model, two separate very similar structures exist. One belongs to the commitment entities and the other to the event entities. It is said that there is mirroring between event and commitment structures [2]. Both structures relate to agent and resource entities. Despite the fact that the REA approach explicitly defines a specific contract entity there is still the open issue of how to create this type of entity which is immaterial in character. Another REA weak point is the absence of a clear state machine declaration. Currently, states are derived from the states of resources during exchange or conversion processes. 
There is an idea of how both ontologies can be utilized in mutual accordance. DEMO is fully aware of everything that happens in the real world with high empirical evidence. Utilizing DEMO on the most generic level would mean having a modeling approach that covers broad modeling abilities and is closer to human beings. The REA modeling approach could be exploited to a higher degree and closely cooperate with the generic level. REA approach would be utilized due to domain specific knowledge and the ability to solve its specific production actions. However, this idea would require simulating the REA model (its coordination part) by the DEMO methodology. The problematic issue relating to this idea is the relatively large variety of the 'give' and 'take' transactions which are interrelated. In addition, these transactions may be delayed in time.

\section{Conclusion}

The aim of the paper was to show and with a simple example demonstrate the similarities and differences between the DEMO and REA concepts of transaction. The stated example illustrates the DEMO approach, which, with simple transactions, can model future commitments and in this way contracted actions. The REA value modeling approach that distinguishes between past, current and future events, and uses an explicitly defined contract entity for this reason. A contract entity contains related commitments and commitments that would be instantiated in exceptional circumstances. The paper also considered the idea of mutual collaboration of these different approaches of business process modeling.

\section{ACKNOWLEDGMENT}

This paper was supported by the grant reference no.SGS14/PrF/2015 provided by Ministry of Education, Youth and Sports of the Czech Republic.

\section{REFERENCES}

[1] G. L. Geerts, and W. E. McCarthy, "The Ontological Foundation of REA Enterprise Information Systems". Paper presented at the Annual Meeting of the American Accounting Association, Philadelphia, PA., 2000.

[2] G. L. Geerts, W. E. McCarthy, "Polocy-Level Specification in REA Enterprise Information Systems". Journal of Information Systems. Vol 20, No. 2, 2006, pp. 37-63. DOI: 10.2308/jis.2006.20.2.37

[3] J. L. G. Dietz, "Enterprise Ontology - Theory and Methodology". Springer-Verlang, 2006.

[4] J. L. G. Dietz, "The Essence of Organization. An Introduction to Enterprise Engineering”. Sapio bv, 2012.

[5] Ch. L. Dunn, O. J. Cherrington, and A. S. Hollander, "Enterprise Information Systems: A Pattern Based Approach". New York: McGraw-Hill/Irwin, 2004.

[6] P. Hruby, "Model-Driven Design Using Business Patterns". SpringerVerlang, 2006.

[7] F. Hunka, and J. Zacek, "Detailed Analysis of REA Ontology", Lecture Notes in Busines Information Processing, Vol. 174, 2014, pp. 61-75. DOI: 10.1007/978-3-319-06505-2

[8] R. Klimek and P. Szwed, "Verification of ArchiMate Process Specification Based on Deductive Temporal Reasoning". Proceedings of the 2013 Federated Conference an Computer Science and Information Systems. pp. 1103-1110.

[9] R. Wendler, "Delelopment of the Organizational Agility Maturity Model". Proceedings of the 2014 Federated Conference on Computer Science and Information Systems. Vol 2, 2014, pp. 1197-1206. DOI: $10.15439 / 2014 \mathrm{~F} 79$ 